Neuroepidemiology 2003;22:366

DOI: $10.1159 / 000072928$

\section{Chemotherapy for High-Grade Glioma}

Sarah Burdett, Lesley Stewart, on behalf of the Glioma Meta-Analysis Trialists Group

\section{Question}

Does giving chemotherapy in addition to surgery and radiotherapy prolong survival in adult patients with high-grade glioma?

\section{Data Source}

Individual patient data systematic review and meta-analysis of 12 randomized controlled trials $[1,2]$. Trials were identified by searching Medline, CancerLit and Embase. These searches were supplemented by hand-searching reference lists of identified trials and bibliographies of relevant books and review articles. A number of trial registers were searched to help identify unpublished, recently closed and ongoing trials. Trialists who supplied their data were also asked to help identify trials. An international collaborative project aimed to collect, validate and reanalyze 'raw' data on individual patients from all relevant randomized controlled trials, obtained from the trialists responsible for the trials. This individual patient data approach permits time-to-event analysis, which is important in malignant glioma where extending survival rather than cure is anticipated. It also allows subgroup analyses to assess whether chemotherapy may be more or less effective for different types of patients.

\section{Patients}

Adult patients with high-grade glioma who had undergone surgery and had not received any prior treatment for any other malignancy likely to interfere with protocol treatments.

\section{Treatment}

All trials included at least one nitrosourea compound given as either single agent or in combination with other drugs. Total radiotherapy doses ranged from 40 to 60 Gy given in 20-35 fractions. The maximum planned delay between surgery and radiotherapy/chemotherapy ranged from 2 to 6 weeks.

\section{Outcome Measures}

Overall survival; progression-free survival.

\section{Quality of the Studies}

Only trials with adequate methods of randomization were included. All data received were checked thoroughly to ensure both the accuracy of the meta-analysis database and the quality of randomization and follow-up. Any queries were resolved and data verified by trial investigator or statistician.

\section{Main Results}

3,004 patients from 12 randomized controlled trials were included (11 published, 1 unpublished). Results show a significant pro-
Table 1. Benefit of chemotherapy in adults with high-grade glioma

\begin{tabular}{llccl}
\hline $\begin{array}{l}\text { Events/ } \\
\text { treatment }\end{array}$ & $\begin{array}{c}\text { Events/ } \\
\text { control }\end{array}$ & HR & $95 \% \mathrm{CI}$ & ASD \\
\hline $\begin{array}{l}\text { Overall survival at 1 year } \\
1,484 / 1,698 \quad 1,175 / 1,306\end{array}$ & 0.85 & $0.78-0.92$ & $6(40 \rightarrow 46 \%)$ \\
\hline $\begin{array}{l}\text { Progression-free survival at 1 year } \\
1,035 / 1,136 \quad 824 / 886 \quad 0.83\end{array}$ & $0.75-0.91$ & $5(18 \rightarrow 23 \%)$ \\
\hline
\end{tabular}

$\mathrm{HR}=$ Hazard ratio; $\mathrm{ASD}=$ absolute survival difference

longation of survival associated with chemotherapy (table 1), with a hazard ratio of $0.85(95 \% \mathrm{CI} 0.78-0.91 ; \mathrm{p}=0.00004)$ or $15 \%$ relative decrease in the risk of death. This is equivalent to an absolute increase in 1-year survival of 6\% (95\% CI 3-9\%) from 40 to $46 \%$ and a 2-month (95\% CI 1-3) increase in survival time from 10 to 12 months. Analysis of progression-free survival, which was based on 8 trials (2,022 patients), gave similar results with a hazard ratio of 0.83 $(95 \%$ CI $0.75-0.91 ; p=0.00008)$ and a $17 \%$ reduction in the risk of progression or death. This is equivalent to an absolute benefit of $5 \%$ at 2 years increasing progression-free survival from 10 to $15 \%$. There was no evidence that the relative effect of chemotherapy varied by age, sex, histology, performance status or extent of resection.

\section{Conclusion}

This small improvement in survival by chemotherapy encourages further study of drug treatment of these tumors.

\section{Comment}

This meta-analysis has shown a clear benefit of chemotherapy of around $6 \%$ at 1 year and an improvement in median survival time of 2 months, increasing median survival from 10 to 12 months. Whether this is of benefit clinically remains open to interpretation. Few of the trials included in this meta-analysis formally measured quality of life in ways that would allow data to be combined. We were therefore unable to assess the quality of the demonstrated prolongation of survival.

\section{References}

1 Glioma Meta-analysis Trialists (GMT) Group: Chemotherapy in adult high-grade glioma: A systematic review and meta-analysis of individual patient data from 12 randomized trials. Lancet 2002;359:1011-1018.

2 Glioma Meta-analysis Trialists Group: Chemotherapy in adult high-grade glioma: A systematic review and meta-analysis of individual patient data from 12 randomized trials; in Cochrane Library, Issue 4, Oxford, 2002. Update Software.

Sarah Burdett

MRC Clinical Trials Unit

222 Euston Road, London NW1 2DA (UK)

E-Mailsb@ctu.mrc.ac.uk

\section{KARGER}

(C) 2003 S. Karger AG, Basel

Fax +4161306 1234

E-Mail karger@karger.ch

www.karger.com
Accessible online at: www.karger.com/ned 